\title{
Use of remote Earth sensing for agro-ecological estimation of soil cover structures and optimisation of agriculture in the forest-steppe zone of the Krasnoyarsk territory
}

\author{
Yuliya V. Gorbunova, Tatyana N. Demyanenko*, and Valentina V. Chuprova \\ Federal State Budget Educational Institution of Higher Education Krasnoyarsk State Agrarian \\ University, 90 Mira Avenue, Krasnoyarsk City, 660049, Russia
}

\begin{abstract}
The possibilities of using data of Earth remote sensing for the construction of a geomorphological profile for the purpose of agroecological assessment of soil cover patterns and subsequent land typification as a basis for the design of adaptive landscape agriculture in the Krasnoyarsk Territory are considered. The geomorphological profile illustrates the features of the relief and regularities in the distribution of soil structures with a certain set of agro-ecological conditions and limiting factors that determine the choice of agro-technologies and farming systems.

Keywords: SRTM data, digital relief model, digital soil map, geomorphological profile (catena), structure of soil cover.
\end{abstract}

\section{Introduction}

Use of the Earth remote sensing (ERS) data is one of current trends in increasing efficiency of agricultural industry. It is caused by the ability of GIS software to solve the tasks of spatial location of cultivable areas with a set of crops corresponding to their fertility. The use of satellite data allows analyzing large areas, revealing migration trends, constructing sets of special maps by certain parameters which characterize various aspects of cultivated land. An outstanding feature of the obtained data is providing possibility for the development of new agro-technologies to optimize agriculture.

To use their landholdings efficiently, agricultural enterprises must be well aware of, first of all, the relief and the soil structure of cultivable areas [1,2]. Relief is the key factor of soil formation; it has a direct influence on the processes flowing in soil stratum, including development of erosion phenomena. Relief determines spatial heterogeneity of topsoil which often leads to allocating various types of soil even within the limits of one farm field. This is why ERS technology is used to solve technological tasks in agriculture. Catena transects, being commonly used as cartographic units, are characterized by the biggest certainty of detecting substance migration flow [3] and topsoil functioning related to it.

\footnotetext{
* Corresponding author: t-demyanen@mail.ru
} 
The aim of this work is to create a geomorphological profile (catena) with the use of ERS data for agro-ecological estimation of soil cover structures and typification of lands.

\section{Objects and methods}

The cultivated land used by ZAO "Iskra", Uzhursky district, Krasnoyarsk territory, has been chosen for research. One field contains a geomorphological profile which represents a catena (centre coordinates: $89,76^{\circ}$ and $55,40^{\circ}$ ) on a convex slope with a steepness about $4,5 \mathrm{o}$ covered by agricultural vegetation. Catena length is $2250 \mathrm{~m}$. The catena ends with a massive degradation.

Three positions, or elementary geochemical landscapes, were allocated, samples of soil profiles were taken, and the main outlines of soil profiles are designated according to the relief conditions on the catena during the on-site inspection. The on-site inspection was preceded by the choice and creation of a geomorphological profile with the use of MapInfoProfessional software. SRTM data were used to explore the data [4]. SRTM (The Shuttle Radar Topography Mission) provides the relief data for almost all the Earth surface obtained by "Space shuttle" spacecraft to create the most complete electronic database of the high definition Earth relief data.

Basing on the space imagery results, the catena position has been worked out, largescale thematic maps titled "Relief" and "Topsoil" have been compiled and superimposed in two layers. To digitize "Relief" map we used the soil map of the farm territory compiled in 1988 by Roszemproject of Krasnoyarsk Vostsibgiprozem Institute in scale 1:25000.

\section{Results and discussion}

ZAO "Iskra" is located in Chulym-Yenisey basin, restricted by the Batenevsky range (the Kuznetsk Alatau spur) in the south and by the Solgon range (the Eastern Sayan spur) in the north. On this territory the relief is considered to be formed under the influence of [5] glacial processes (displayed on the mountain summits), erosional processes (associated with the ground layer of mountains and ranges, accumulative when declining). A distinctive feature of the present-day relief of the basin is heterogeneity [6]. Figure 1 represents the digital relief model of the area. It is apparent that the distinctive relief forms of the southern part of the area are hilly uplifts, hummocks, and monoclinal hills (cuestas). Flat-plain relief of intercuesta degradation is noted in the central part, and hilly-plain relief with ridges and hills in the northern part. Slope location and "mountainous" framing (720-815 m. above sea level) of the area account for the development of denudation, diluvial, and erosional accumulative processes.

The climate of the area is very continental (Ivanov index of continentality -210 ) and semihumid. According to the figures provided by the Uzhur weather station, the average annual precipitation equals to the average annual evapotranspiration, and it amounts to $464 \mathrm{~mm}$; the distribution of precipitation throughout the year is extremely inhomogeneous, only $17 \%$ of the annual precipitation occurs from November to April, the biggest amount of precipitation occurs in July and September (44\% of the annual amount), often as showers which causes intense erosion on the slopes. Humidity factor of 1.0 characterizes the type of water regime as occasionally percolative. Selyaninov hydrothermal index is 1.3 which corresponds to sufficient humidity. Growing season is 154 days, but effective temperatures become stationary only on the 27 th of May, thus the period of active vegetation amounts to 109 days. 

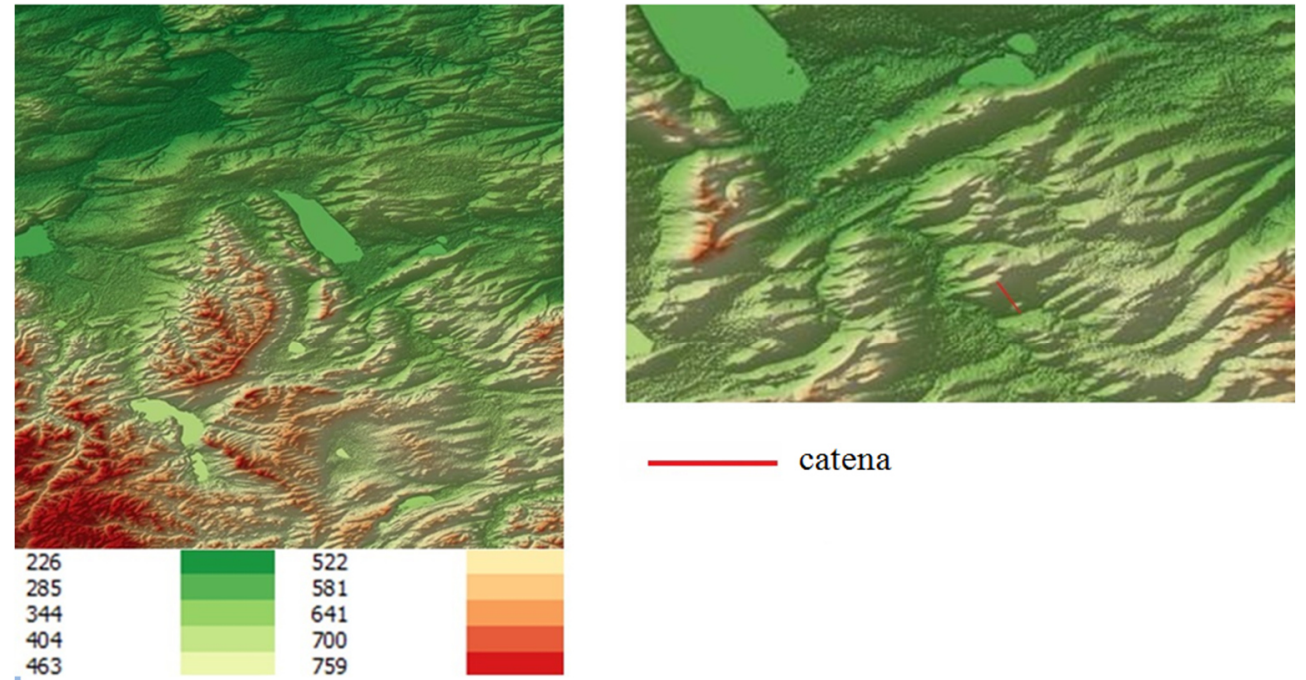

catena

Figure 1 - Digital relief model of ZAO "Iskra" area (map key - altitude above the sea level, $m$.)

Cultivated lands are allocated according to the relief analysis, are differentiated depending on the soil parent material composition, ground water level, topsoil heterogeneity, physical and chemical soil characteristics, the degree of plowing of humic accumulative horizons, presence or absence of erosional processes. Positional distribution of cultivated lands is determined by the peculiarities of mesorelief and microrelief which widely vary over the different land areas of ZAO "Iskra".

Further, using the experience of field (on-site) inspection and certain experience of large-scale mapping of relief and topsoil structure, it is possible to specify allocated positions of elementary landscape, or even to divide it into additional categories. It is obvious now that the catena is remarkable for its complexity and accentuation both on lithological features and on migration flows which cause domination of denudation over erosion, or, vice versa, domination of erosional processes. More detailed division also implies compilation of special maps by certain parameters of migration processes.

The geochemical positions emphasized at this stage of the research are: eluvial denudation landscape (I), transitional landscape (II), eluvial accumulative landscape (III). They can be considered as microcatenas of a lower order (Fig. 2). The superimposed image of the layers titled "Relief" and "Soil map" of the cultivable area of ZAO "Iskra" (Fig.3) shows that soil-forming process runs under harsh conditions of rock weathering, short annual duration of soil-forming process, shallow invasion of soil weathering into sedimentation mass. The catena slope is characterized by bedding of soil parent materials close to the surface, as indicated by the abundance of coarse waste. Agricultural use of this territory causes intensive anthropogenesis which launches erosion accumulative processes. It is shown up in wide spread of shortened humus horizon soil and other erosional features. 


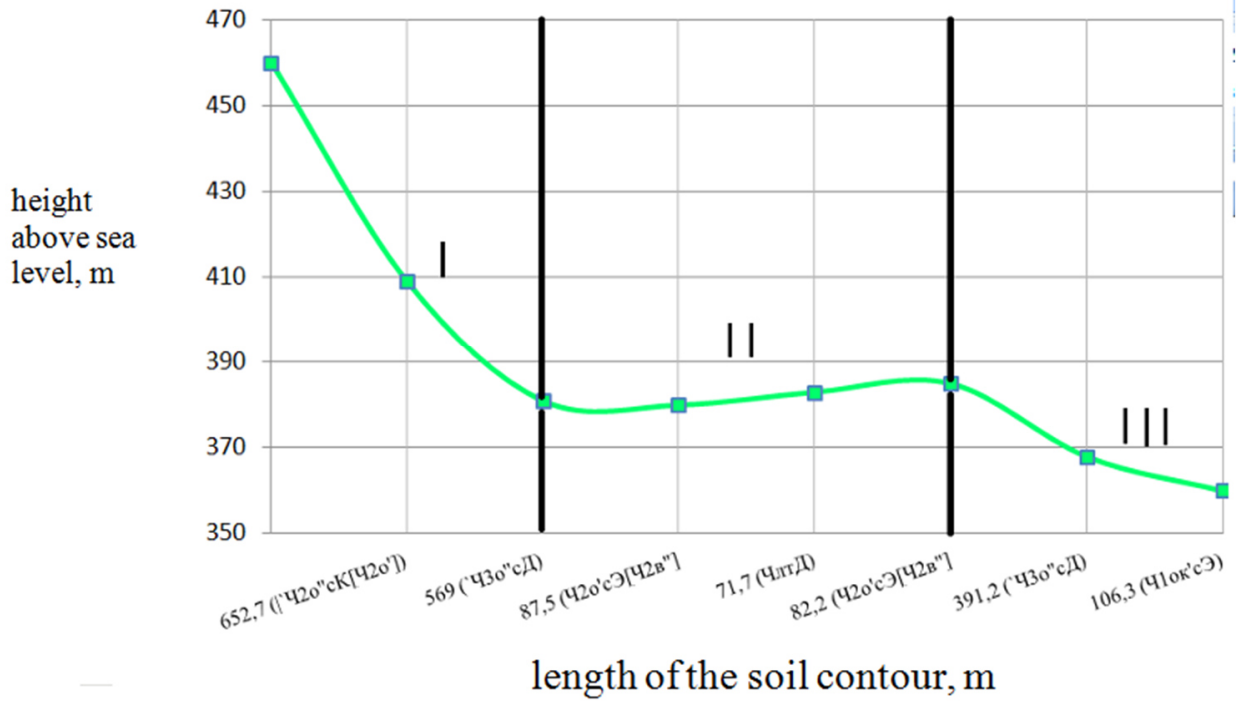

Figure 2 - Geomorphological profile of ZAO "Iskra"

$42 o^{\prime \prime} \mathrm{cK}\left[42 o^{\prime}\right]$ - ordinary medium-humic, middle-deep, slightly eroded chernozem along with ordinary middle-deep chernozem (10-25\%);

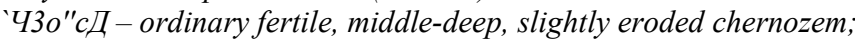

$42 o^{\prime} c Э\left[426^{\prime \prime}\right]$ - ordinary medium-humic, shallow chernozem along with leached, medium-humic, middle-deep chernozem (10-25\%);

ЧлтД - meadow chernozemic soil along with chernozemic meadow soil;

$41 \kappa^{\prime}$ 'Э - ordinary calcium low-humic, shallow chernozem,
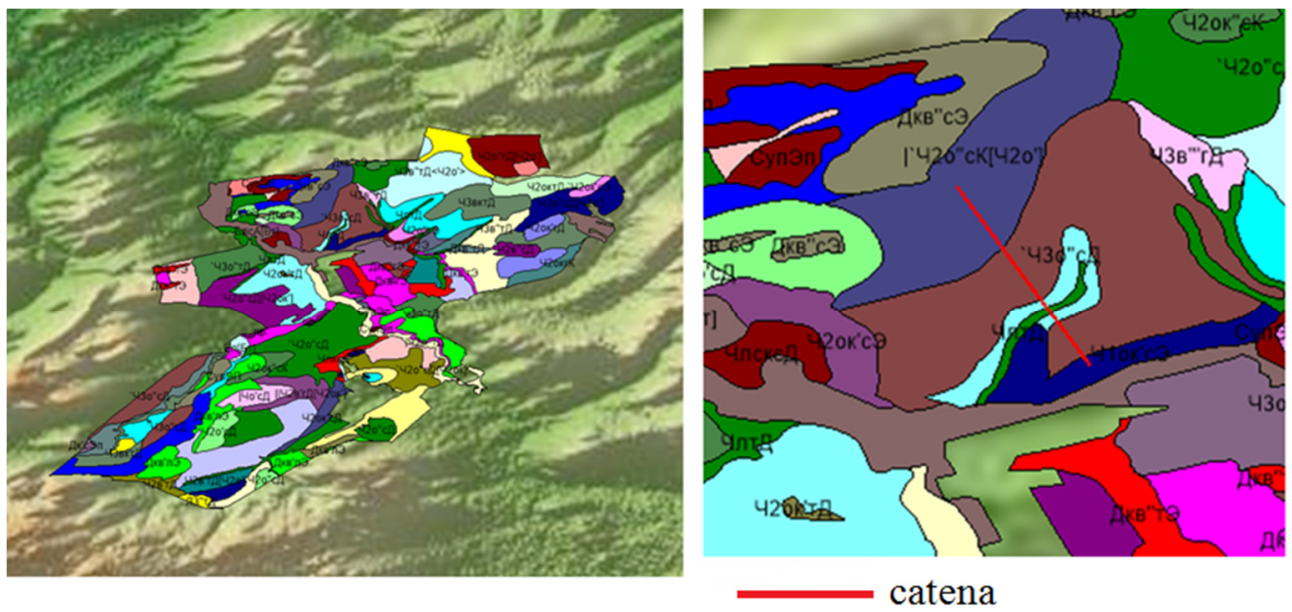

Figure 3 - Superimposition of two layers "Relief" and "Soil Map"of ZAO "Iskra" cultivated area

Lot I - the most elevational summit part of the catena with altitude marks from 380 to 460 metres above sea level; it has quite a high slope angle contributing to mainly horizontal material migration with atmospheric precipitation. This flow links the lot to the underlying catena positions. Vertical precipitation material inflow provides for the functioning of the soil profile of the ordinary shallow and middle-deep eroded chernozem complex. The top part of the soil profile is characterized by the high depth variability of the humus horizon 
and carbonate illuviation signs. The lower part of the profile is noted for its mottled redbrown colour, genetically bound to Devonian rocks. The texture of the whole soil profile is quite homogeneous, middle-loamy.

Lot II occupies the central part of the slope (380-385 metres above sea level), has a slight slope angle and is characterized by a gently concave surface with multiple microdepressions. Here atmospheric moistening prevails, erosional processes dominate, causing impoverishment of soil in humus and fertilizer elements. In depressed relief forms carbonate leaching is noticed. This is why the soil background is formed mainly by ordinary and 10-25\%-leached types of chernozem. Land runoff development in this part of the catena is caused by the water flowing down from the surface of the overlying (position I) steep slope of the cultivated land.

Lot III of the geomorhological profile is characterized by a significant slope and ends in the valley part of the territory. Absolute elevation is 385-360 metres above sea level. Atmospheric moisturizing, often combined with ground water moisturizing. Mixed material migration continues here: vertical, influenced by ground water in microdepressions. Eluvial accumulative processes take place in this lot which causes bigger heterogeneity of the soil structure. The aggregate of automorphous and semiterrestrial regimes contributed to the formation of ordinary calcium shallow chernozems on local microhills and complex variations of ordinary deep meadow chernozemic and chernozemic meadow soils on microdepressions.

Thus, the soil massive of the catena is characterized by spatial heterogeneity of agroecological conditions and various fertility limiting features. The traditional approaches to agro-ecological estimation and land type grouping rely upon the studying and mapping of the factors which limit agriculture [8]. At this stage of the research only relief has been viewed as a factor of agro-ecological differentiation of the topsoil. The immediate task is to study indicational characteristics on various positions of the catena to develop digital maps of limiting features and agro-ecological types of soil.

\section{Conclusion}

As a conclusion, it should be pointed out that the use of the Earth remote sensing data opens up new opportunities of studying catena peculiarities of cultivated lands to estimate their agro-ecological state. Through the example of the catena of the cultivated land in Uzhursky district it has been proved that complex slope relief pattern leads to domination interchange of eluvial and accumulative processes on various parts of the cultivated area, being influenced by erosional processes and other fertility-limiting features. That is why in such areas it is necessary to allocate corresponding types of land and to take them into consideration when elaborating adaptive landscape farming systems, protecting and raising soil fruitfulness.

\section{References}

1. Vlasenko A.N., Dobrovolskaya N.I., Semendyaeva N.V. Agro-ecological estimation and typification of lands as the basic element for projecting adaptive landscape farming: a guideline, GNU SibNIIZHSH. - Novosibirsk, 2011, - 55 p.

2. Kudryashova S.Ya., Ditz L. Yu., Chichulin A. V., Chumbaev A.S., Miller G. F., Bezborodova A.N. Ecological - Geographical Aspects of Soil Complex Types Allocation at the Ukok Plateau Using Remote Sensing Studies // Siberian Ecological Journal, 2012, No. 5, pp. 703-710

3. Bogdanova M.D., Gerasimova M. I., Gavrilova I.P. An experience of large-scale mapping of the main objects of the geochemistry of landscape // Geochemistry of 
landscapes and geography of soils. A report at the national conference, Moscow, 4-6 April, 2012. M.: Faculty of Geography MSU, 2012, pp. 64-66.

4. Onkov I.V. Accuracy estimation for SRTM altitudes for orthotransforming high definition space images // Geomatics. 2011, No.3. Pp. 40-46

5. Kallas E.V. Humic profiles of lake basins of Chulym-Yenisey basin. Novosibirsk: Gumanitarnie nauki, 2004. - 170 p.

6. Tanzynbaev M.G. Soils of Khakassia. - Novosibirsk, 1993. - 300 p.

7. Weather archive in Uzhur [electronic resource] - URL: https://rp5.ru/Weather_archive in_Uzhur

8. Sorokina N.V., Kozlov D.N. Methods of digital cartography in agro-ecological land estimation // Digital soil cartography: theoretical and experimental studies. M.: V.V. Dokuchaev Soil Science Institute. 2012. - Pp. 140-154. 